\title{
Time of Maximum Observed Excretion Rate
}

National Cancer Institute

\section{Source}

National Cancer Institute. Time of Maximum Observed Excretion Rate. NCI Thesaurus.

Code C85823.

TThe midpoint of a collection interval associated with the maximum observed excretion rate. 